\title{
Mismatch between older adult's expectation and smartphone user interface
}

\begin{abstract}
Smartphones have become ubiquitous communication tools for everybody, including older adults to stay connected with their family and access to information. However, mobile operators and developers mainly target the youngster cohort in mobile industry. With the rising ageing population, smartphone user interface and some mobile apps are not designed to cater the needs of older adults. This could hinder them from fully utilizing the smartphone functions and its services. A mobile-user interaction study using mixed-methods (questionnaire, interview and observation) was conducted to examine usability and user interface design issues of smartphone and mobile apps among 80 older adults in Malaysia. Four tasks design were 'making voice calls', 'using phonebook', 'installing a mobile app from GooglePlay Store', and 'using WhatsApp'. The results were analysed both quantitatively (for usability evaluation) and qualitatively (for interviews and observation). The usability result revealed that the 'voice call' task had the highest success task completion rate (83.44\%), followed by 'phonebook' $(70.16 \%)$, 'mobile app download'(63.13\%) and 'using WhatsApp' (60.42\%). Three themes were emerged from the qualitative thematic analysis, which showed a mismatch between older adults' expectation and smartphone user interface. A majority had never downloaded a mobile app before, and they had problems down loading it from Play Store. They perceived the Play Store feature as a place for children playing games. To close the discrepancy between user expectation and mobile design, the mobile designers and developers are required to consider the older adults' needs for better usability of smartphone user interface design.
\end{abstract}

Keyword: Older adults; Mismatch expectation; Smartphone user interface; Usability; Design issues 